\title{
The Asymmetric Outflow of RS Ophiuchi
}

\author{
S. Mohamed ${ }^{1}$, R. Booth ${ }^{2}$, and Ph. Podsiadlowski ${ }^{2}$ \\ ${ }^{1}$ Argelander Institut für Astronomie, Auf dem Hügel 71, Bonn D-53121, Germany \\ email: smohamed@astro. uni-bonn.de \\ ${ }^{2}$ Department of Astrophysics, University of Oxford, Oxford OX1 3RH \\ email: richard.booth@astro.ox.ac.uk, podsi@astro.ox.ac.uk
}

\begin{abstract}
RS Ophiuchi (RS Oph) is a symbiotic binary consisting of a hot white dwarf accreting from the slow, dense stellar wind of a cool, red giant companion. The system belongs to, and is one of the best studied examples of, an even smaller subclass of binaries known as recurrent novae in which the white dwarf undergoes repeated thermonuclear outbursts. We present 3D smoothed particle hydrodynamics (SPH) models of mass transfer from the red giant to the white dwarf, followed by a nova outburst. We show that the outflow in the former is strongly concentrated towards the binary orbital plane. The nova ejecta is thus constrained in the equatorial directions, resulting in a bipolar outflow. The white dwarf in RS Oph is thought to be close to the Chandrasekhar mass, making the system a likely Type Ia supernova candidate. We discuss the role that such a highly structured circumstellar medium will play in the evolution of the supernova remnant.
\end{abstract}

Keywords. hydrodynamics — binaries: symbiotic - stars: novae, cataclysmic variables, individual (RS Oph), supernovae

\section{Introduction}

Despite decades of theoretical and observational studies, there is still no definitive identification of the progenitors of Type Ia supernovae (SNe Ia). It is widely agreed, however, that SNe Ia result from the thermonuclear disruption of a carbon-oxygen white dwarf (C-O WD) close to the Chandrasekhar mass, $\sim 1.38 \mathrm{M}_{\odot}$. There are currently two main scenarios in which the WD can achieve such a mass; in the "single-degenerate (SD)" channel the WD accretes material from a non-degenerate companion, whereas the "double-degenerate (DD)" channel involves a merger with another WD. In this work, we present a preliminary hydrodynamical study of a well-known possible SD progenitor, RS Ophiuchi (RS Oph).

RS Oph is a symbiotic binary consisting of a red giant (RG) and a WD. The RG is losing mass via a dense stellar wind, part of which accumulates on the surface of the WD. When this material gets hot enough to ignite hydrogen, a thermonuclear runaway, or nova, results. The system also belongs to a smaller subclass of novae known as recurrent novae, undergoing explosive outbursts on the order of decades in 1898, 1933, 1958, 1967, 1985, 2006, and possibly in 1907 and 1945 (see Anupama and Mikolajewska, this volume). The short recurrence period, high accretion rate, and low ejecta masses are consistent with a WD that is close to the Chandrasekhar limit (Yaron et al. 2005).

The RS Oph NaI D lines before, during and after the nova outburst show similar variations to those discovered in some SNe Ia, e.g. SN 2006X, SN 2007le (Patat et al. 2007, Simon et al. 2009, Sternberg et al. 2011), providing strong evidence for a further connection between RS Oph and these SNe Ia (Patat et al. 2011, F. Patat, this volume). The variations are attributed to the changing ionization state of $\mathrm{Na}$ in the circumstellar 

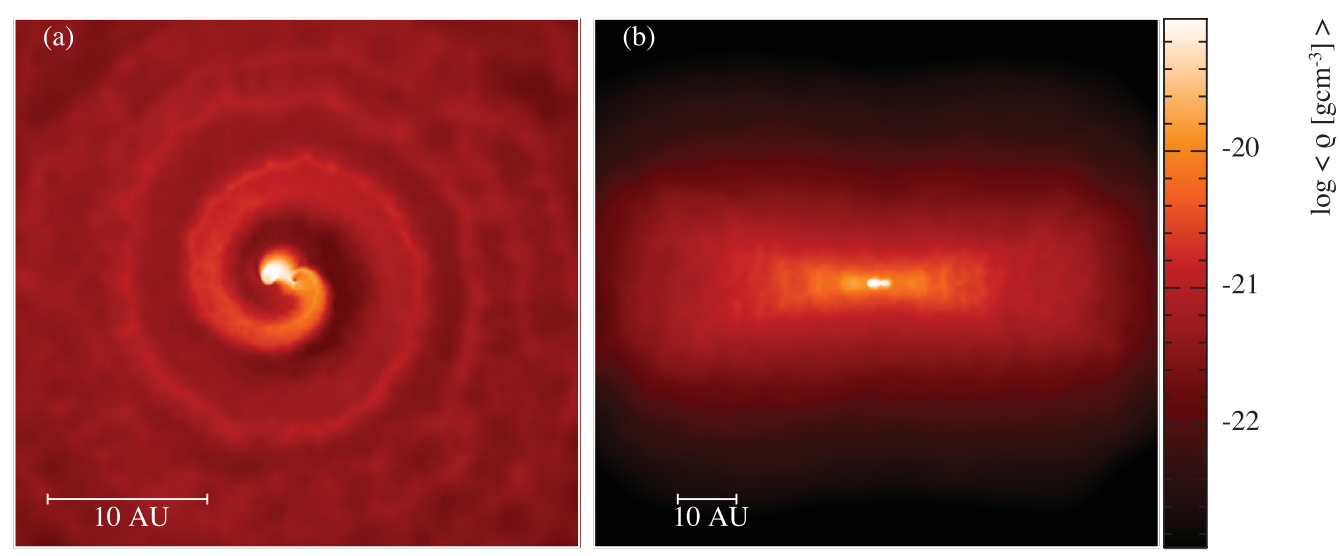

Figure 1. The circumstellar structure produced during the RS Oph mass-transfer phase. The projected gas density is shown with inclination angles $0^{\circ}$ (face-on) [a] and $90^{\circ}$ (edge-on, i.e. perpendicular to the orbital plane) $[\mathrm{b}]$.

environment as it interacts with the nova/supernova radiation and ejecta and then later cools and recombines.

\section{The Model}

In order to better understand the origin of these $\mathrm{NaI} \mathrm{D}$ line variations, we model the interaction of a nova with a circumstellar medium (CSM) produced by a symbiotic binary. We utilize smoothed particle hydrodynamics (SPH), a Lagrangian method particularly suited to studying hydrodynamical flows with arbitrary geometries (for detailed reviews, see Rosswog 2009, Springel 2010, Price 2012). Throughout this study we use the GADGET-2 SPH code (Springel 2005) which we have modified to include binary motion, stellar winds, mass accretion and molecular and atomic radiative cooling (see Mohamed 2010, Mohamed et al. 2011).

\subsection{The Binary Mass-transfer Phase: Equatorial Outflows}

The binary consists of a $0.8 \mathrm{M}_{\odot} \mathrm{RG}$ and a $1.38 \mathrm{M}_{\odot}$ WD separated by $a=1.48 \mathrm{AU}$ (based on parameters derived by Brandi et al. 2009 for RS Oph). Particles are injected at the surface of the RG which we assume is 0.8 times the Roche-lobe radius. The mass-loss rate and velocity of the outflow are $\dot{M}_{\text {loss }}=1 \times 10^{-7} \mathrm{M}_{\odot} \mathrm{yr}^{-1}$ and $v_{\text {wind }}=$ $20 \mathrm{~km} \mathrm{~s}^{-1}$, respectively. The radius of the WD cannot be resolved in the simulations, thus the accretion radius, the boundary where particles are removed from the flow, is set to $\sim 0.01 \mathrm{AU}$. The CSM produced by the binary interaction is highly aspherical; the binary motion results in the formation of an Archimedes spiral shock (Figure 1a) which is focused by the gravity of the massive WD resulting in an equatorial outflow (Figure 1b).

\subsection{The Nova Outburst: Bipolar Outflows}

We model the nova as a spherically symmetric explosion centered on the WD with mass, velocity, and outburst energy of the ejecta of $M_{\text {ejecta }}=2 \times 10^{-7} \mathrm{M}_{\odot}, v_{\text {ejecta }}=4,000$ $\mathrm{km} \mathrm{s}^{-1}$, and $E_{\text {ejecta }}=10^{43} \mathrm{ergs}$, respectively (Yaron et al. 2005, Bode 2010, and references therein). The nova ejecta, constrained in the orbital plane by the dense CSM produced during the mass-transfer phase, expands more slowly in that direction while the flow is largely unrestricted in the polar directions. The result is a strongly bipolar outflow (see Figure 2 [right]) as was observed for RS Oph with HST (Bode et al. 2007, Ribeiro et al. 

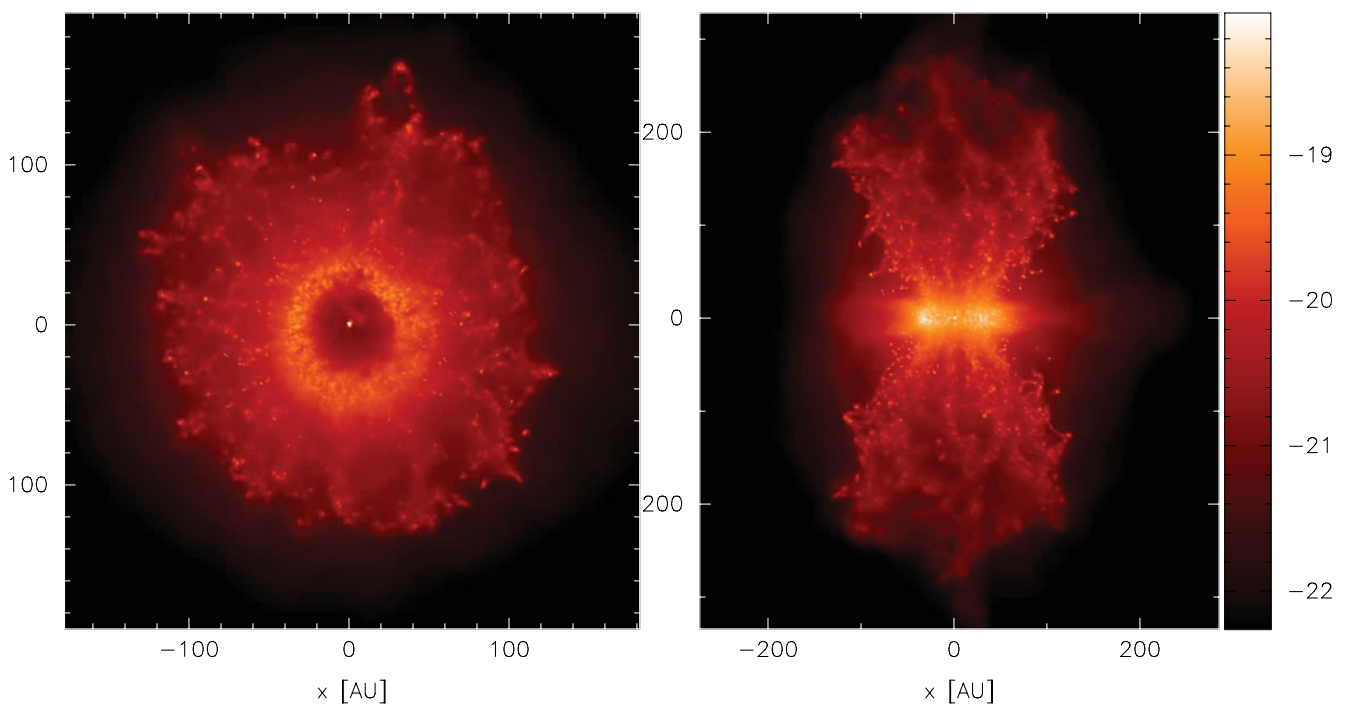

Figure 2. The projected gas density $\left(\log <\rho\left[\mathrm{g} \mathrm{cm}^{-3}\right]>\right)$ produced by the interaction of a nova with the RS Oph circumstellar medium viewed with inclination angles $0^{\circ}$ (face-on) [left] and $90^{\circ}$ (edge-on) [right].

2009) and found in previous studies (e.g., Walder et al. 2008). In our models, as the nova ejecta sweeps up the aspherical CSM, strong instabilities form knots and clumps which may be important density enhancements required to produce the Na I D line variations. The morphology of the nova shell when viewed face-on (see Figure 2 [left]) is very similar to that of clumpy shell of T Pyx (Shara et al. 1997) $\dagger$.

\subsection{The CSM-SN Interaction}

In Figure 3 we show the results of the W7 model (Nomoto et al. 1984) for SN ejecta, with an explosion energy of $1.4 \times 10^{51} \mathrm{erg}$, interacting with constant density and wind-blown circumstellar environments. The remnant structure consists of a forward shock (shocked CSM) and a reverse shock (shocked ejecta) separated by a contact discontinuity. The ejecta also drives a shock through the companion, stripping off a large portion of its envelope. A low density cavity forms in the region shadowed by the companion, and Rayleigh-Taylor and Kelvin-Helmholtz instabilities disrupt the high density ejecta around the edge of the hole causing it to slowly close (see also García-Senz et al. 2012). While the global morphology of the remnants is similar, the different properties of the CSM do leave an imprint on the remnant, largely in terms of the mass swept up by the SN shell at a given time; the ratio of swept up mass to SN mass are 2.5 and 0.1 for the constant density and wind CSM models, respectively (Booth et al., in prep.).

\section{Conclusions}

The extensive and detailed multi-wavelength observations of RS Oph make it an ideal laboratory to test our theories of both mass transfer and the interaction of fast ejecta with a pre-existing CSM. The results of our 3D high resolution simulations show that the nova ejecta interacts with a highly aspherical outflow resulting in the formation of a clumpy, bipolar outflow. Our preliminary analysis suggests that lines of sight towards

$\dagger$ Chesneau et al. (2011) recently found that the 2011 outburst was best fitted with a bipolar outflow and inclination angle of $15^{\circ}$. 

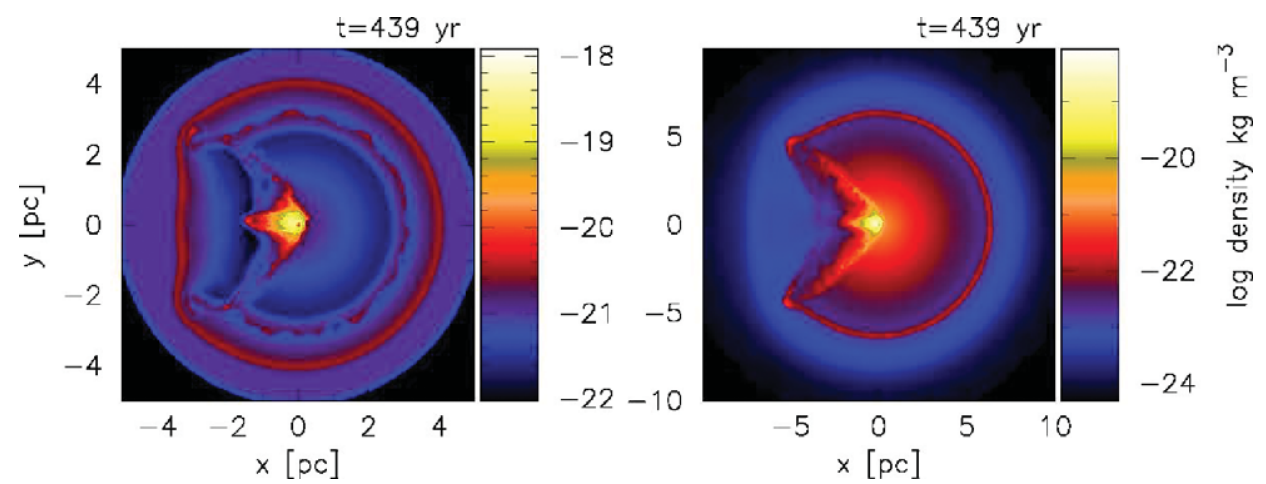

Figure 3. Density cross-sections of the remnant structures produced by the interaction of a Type Ia supernova with different circumstellar environments; [Left] CSM with constant $10^{-24}$ $\mathrm{g} \mathrm{cm}^{-3}$ density; [Right] Wind-blown CSM (i.e. $\rho \propto r^{-2}$ ) with $\dot{M}_{\text {loss }}=10^{-6} \mathrm{M}_{\odot} \mathrm{yr}^{-1}$ and wind velocity of $50 \mathrm{~km} \mathrm{~s}^{-1}$.

the orbital plane have sufficient column density to account for the Na I D line variations. A detailed study of the $\mathrm{Na}$ lines after several nova cycles will be carried out. Moreover, studies of the interaction of a SN with the more complex, bipolar nova CSM are also currently underway.

\section{Acknowledgements}

The rendered figures in this paper were made using a modified version of the SPLASH visualization toolkit (Price 2007).

\section{References}

Bode, M. F., Harman, D. J., O'Brien, T. J., Bond, H. E., et al. 2007, ApJL, 665, L63

Bode, M. F. 2010, Astron. Nachr., 331, 160

Brandi, E., Quiroga, C., Mikołajewska, J., Ferrer, O. E., \& García, L. G. 2009, AE\&A, 497, 815

Chesneau, O., Meilland, A., Banerjee, D. P. K., Le Bouquin, J.-B., et al. 2011, A\&A, 534, L11

García-Senz, D., Badenes, C., \& Serichol, N. 2012, ApJ, 745, 75

Mohamed, S. 2010, Ph.D. thesis, Univ. Oxford

Mohamed, S., Mackey, J., \& Langer, N. 2011, ArXiv e-prints

Nomoto, K., Thielemann, F.-K., \& Yokoi, K. 1984, ApJ, 286, 644

Patat, F., Chandra, P., Chevalier, R., Justham, S., et al. 2007, Science, 317, 924

Patat, F., Chugai, N. N., Podsiadlowski, P., Mason, E., et al. 2011, A\& A, 530, A63

Price, D. J. 2007, PASA, 24, 159

Price, D. J. 2012, Journal of Computational Physics, 231, 759

Ribeiro, V. A. R. M., Bode, M. F., Darnley, M. J., Harman, D. J., et al. 2009, ApJ, 703, 1955

Rosswog, S. 2009, NewAR, 53, 78

Shara, M. M., Zurek, D. R., Williams, R. E., Prialnik, D. et al. 1997, AJ, 114, 258

Simon, J. D., Gal-Yam, A., Gnat, O., \& Quimby, R. M. 2009, ApJ, 702, 1157

Springel, V. 2005, MNRAS, 364, 1105

Springel, V. 2010, ARA\&A, 48, 391

Sternberg, A., Gal-Yam, A., Simon, J. D., Leonard, D. C. et al. 2011, Science, 333, 856

Walder, R., Folini, D., \& Shore, S. N. 2008, A\&A, 484, L9

Yaron, O., Prialnik, D., Shara, M. M., \& Kovetz, A. 2005, ApJ, 623, 398 